\title{
Mechanical stress is associated with right ventricular response to pulmonary valve replacement in patients with repaired tetralogy of Fallot
}

\author{
Dalin Tang, PhD, FAHA, ${ }^{\mathrm{a}, \mathrm{b}}$ Chun Yang, MS, ${ }^{\mathrm{b}, \mathrm{c}}$ Pedro J. del Nido, MD, ${ }^{\mathrm{d}}$ Heng Zuo, MS, ${ }^{\mathrm{b}}$ \\ Rahul H. Rathod, MD, ${ }^{\mathrm{e}}$ Xueying Huang, PhD, ${ }^{\mathrm{b}, \mathrm{f}}$ Vasu Gooty, MD, ${ }^{\mathrm{e}}$ Alexander Tang, BA, \\ Kristen L. Billiar, PhD, FASME, ${ }^{\mathrm{g}, \mathrm{h}}$ Zheyang $\mathrm{Wu}, \mathrm{PhD},{ }^{\mathrm{b}}$ and Tal Geva, $\mathrm{MD}^{\mathrm{e}}$
}

\begin{abstract}
Objective: Patients with repaired tetralogy of Fallot account for a substantial proportion of cases with late-onset right ventricular failure. The current surgical approach, which includes pulmonary valve replacement/insertion, has yielded mixed results. Therefore, it may be clinically useful to identify parameters that can be used to predict right ventricular function response to pulmonary valve replacement.
\end{abstract}

Methods: Cardiac magnetic resonance data before and 6 months after pulmonary valve replacement were obtained from 16 patients with repaired tetralogy of Fallot ( 8 male, 8 female; median age, 42.75 years). Right ventricular ejection fraction change from pre- to postpulmonary valve replacement was used as the outcome. The patients were divided into group $1(\mathrm{n}=8$, better outcome) and group $2(\mathrm{n}=8$, worst outcome). Cardiac magnetic resonance-based patient-specific computational right ventricular/left ventricular models were constructed, and right ventricular mechanical stress and strain, wall thickness, curvature, and volumes were obtained for analysis.

Results: Our results indicated that right ventricular wall stress was the best single predictor for postpulmonary valve replacement outcome with an area under the receiver operating characteristic curve of 0.819. Mean values of stress, strain, wall thickness, and longitudinal curvature differed significantly between the 2 groups with right ventricular wall stress showing the largest difference. Mean right ventricular stress in group 2 was $103 \%$ higher than in group 1.

Conclusions: Computational modeling and right ventricular stress may be used as tools to identify right ventricular function response to pulmonary valve replacement. Large-scale clinical studies are needed to validate these preliminary findings. (J Thorac Cardiovasc Surg 2016;151:687-94)

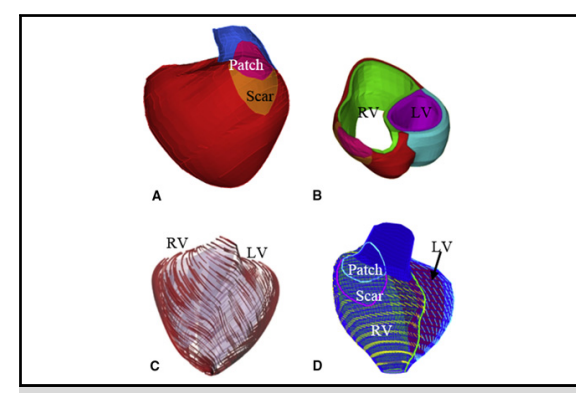

CMR-based model construction process: image, segmentation, 3-dimensional geometry, and fiber orientation.

Central Message

RV stress was the best predictor among 6 morphologic and mechanical factors and may be used to optimize post-PVR outcome.

Perspective

Current PVR procedures for patients with TOF have mixed results. Post-PVR outcome is highly unpredictable. CMR-based models may be used to identify potential predictors for optimized PVR surgical design and better postPVR outcome. Our initial results indicated that RV stress was the best predictor among 6 factors considered. Large-scale studies are needed to confirm our findings.

See Editorial Commentary page 695.

See Editorial page 623.

\footnotetext{
From the ${ }^{\text {a School }}$ of Biological Sciences and Medical Engineering, Southeast University, Nanjing, China; ' Mathematical Sciences Department, Worcester Polytechnic Institute, Worcester, Mass; ' China Information Technology Designing \& Consulting Institute Co, Ltd, Beijing, China; ${ }^{\mathrm{d}}$ Department of Cardiac Surgery, Boston Children's Hospital, Department of Surgery, Harvard Medical School, Boston, Mass; 'Department of Cardiology, Boston Children's Hospital, Department of Pe-

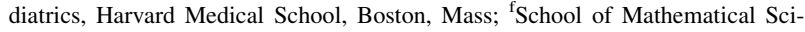
ences, Xiamen University, Xiamen, Fujian, China; ${ }^{\mathrm{g}}$ Department of Biomedical Engineering, Worcester Polytechnic Institute, Worcester, Mass; and ${ }^{\mathrm{h}}$ Department of Surgery, University of Massachusetts Medical School, Worcester, Mass.

This research was supported in part by National Institutes of Health (NIH)-1R01-HL 089269 (P.J.d.N., D.T., T.G.), NIH-HL63095 (P.J.d.N.), NIH/National Heart, Lung,
}

and Blood Institute 5P50HL074734 (T.G.; Co-Investigator P.J.d.N.), and the National Sciences Foundation of China 11171030 and 81571691.

D.T., C.Y., P.J.d.N., and T.G. contributed equally to this article.

Received for publication April 6, 2015; revisions received Sept 2, 2015; accepted for publication Sept 26, 2015; available ahead of print Nov 6, 2015

Address for reprints: Dalin Tang, PhD, FAHA, School of Biological Science \& Medical Engineering, Southeast University, 2 Si Pai Lou, Nanjing, China 210096 (E-mail: dtang@wpi.edu).

$0022-5223 / \$ 36.00$

Copyright (C) 2016 by The American Association for Thoracic Surgery

http://dx.doi.org/10.1016/j.jtcvs.2015.09.106 

Abbreviations and Acronyms
$\mathrm{CMR}=$ cardiac magnetic resonance
$\mathrm{EF}=$ ejection fraction
$\mathrm{LV}=$ left ventricle, left ventricular
PVR = pulmonary valve replacement
ROC $=$ receiver operating characteristic
$\mathrm{RV}=$ right ventricle, right ventricular
$\mathrm{RVOT}=$ right ventricular outflow tract
TOF $=$ tetralogy of Fallot
$\mathrm{WT}=$ wall thickness

Supplemental material is available online.

Survival of patients with tetralogy of Fallot (TOF) has steadily increased since the introduction of open surgery, with operative mortality currently less than $2 \%$. $^{1}$ Survival past the first 2 decades of life also has improved, with recent reports showing a 30 -year survival of approximately $90 \%{ }^{2}$ Since this operation was first performed in the mid-1950s, a conservative estimate projects that the number of survivors of TOF repair in the United States exceeds 100,000 and increases by 3000 to 4000 patients every year. ${ }^{3}$ As a result of the surgical reconstruction of the right ventricular (RV) outflow tract and other operative sequelae, patients are exposed to chronic pulmonary regurgitation that leads to progressive RV dilatation and dysfunction. The current surgical approach to address chronic pulmonary regurgitation includes pulmonary valve replacement (PVR)/insertion with or without RV remodeling. However, although most patients demonstrate a variable degree of decrease in RV size, many do not experience an improvement in RV function and some show a decline after PVR. ${ }^{3-9}$ Facilitated by the advent of cardiac magnetic resonance (CMR) imaging,, 10 data from the INDICATOR cohort have demonstrated that preoperative RV ejection fraction (EF) is an independent predictor of death and sustained ventricular tachycardia in this population. ${ }^{11}$

Recent advances in computational modeling, methods, and computer technology have facilitated the use of computer-simulated procedures to aid clinical decisionmaking, potentially replacing empirical and often risky clinical experimentation to examine the efficiency and suitability of various reconstructive cardiac procedures. There are recent reviews of the modeling development, especially CMR-based ventricle modeling. ${ }^{12-14}$ In our previous articles, patient-specific CMR-based computational RV and left ventricular (LV) models with fluid-structure interactions were introduced to assess outcomes of various RV reconstruction techniques with different scar tissue trimming and patch sizes. ${ }^{15-18}$ These investigations on patients with TOF undergoing PVR have focused on surgical remodeling of the right ventricular outflow tract (RVOT) to improve functional recovery of the RV by removing or reducing the noncontracting tissue (scar and patch) in the outflow tract free wall. ${ }^{19}$ In our previously reported randomized clinical trial of surgical remodeling of the RVOT in 64 patients with repaired TOF undergoing PVR, we found no significant difference between groups in change in RV EF from pre- to 6 months post-PVR. ${ }^{19}$ However, it has remained unclear why some patients experienced an improvement in RV EF and RV function deteriorated in others.

In this study, 3-dimensional computational RV/LV models were constructed for 16 patients with TOF based on their patient-specific pre-PVR CMR data, material parameters chosen to match CMR data, and cathetermeasure pressure conditions. RV stress, strain, volume, ventricular wall thickness (WT), and longitudinal and circumferential curvatures were obtained and used to identify morphologic or mechanical stress/strain markers that may be associated with improved RV function after PVR.

\section{MATERIALS AND METHODS \\ Patients}

The Boston Children's Hospital Committee on Clinical Investigation approved the study. CMR data before and 6 months after PVR were obtained from 16 patients with TOF ( 8 male, 8 female; median age, 42.75 years) (Table E1) who were previously enrolled in our RV surgical remodeling trial. ${ }^{19}$ For this analysis, we selected the 8 best (group 1) and 8 worst (group 2) responders on the basis of their change in RV EF from pre- to post-PVR. RV EF was chosen because of its strong association with adverse clinical outcomes in patients with repaired TOF. Demographic information, RV volumes, pressure conditions, and EF before and after PVR are summarized in Table E1.

\section{Data Acquisition and Modeling}

Data acquisition and modeling procedures have been described, ${ }^{12,15-17}$ and the details are omitted in the current article to avoid repetition. Some key points are summarized next. CMR studies were performed with 1.5 Tesla scanners (GE Medical Systems, Milwaukee, Wis; and Philips Healthcare, Best, The Netherlands). For each cine MR acquisition, 30 frames per cardiac cycle were reconstructed. The location and extent of the RVOT patch were determined on the basis of cine imaging and delayed-enhancement CMR, and were subsequently confirmed by surgical inspection at the time of PVR. Three-dimensional RV/LV geometry and computational meshes were constructed as previously described (Figure 1). ${ }^{15-17}$

Because tissue mechanical properties are essential for computational ventricular modeling, on the basis of the methods of Sacks and Chuong, ${ }^{20}$ Billiar and Sacks, ${ }^{21}$ and Humphrey, ${ }^{22}$ we generated the first complete biaxial mechanical data set for ventricular tissues using a cadaveric normal human heart sample (Figure E1). A detailed description of the custom biaxial testing device and method has been published. ${ }^{20,21}$ We chose parameter values in our modified Mooney-Rivlin model to fit our direct measurement of biaxial stress-strain data. It should be noted that the ex vivo biaxial testing data were used to support the choice of our material model, that is, the modified anisotropic Mooney-Rivlin model (equations are given later) is able to represent ventricle tissue anisotropic material properties. The parameter values in the material model for each patient were determined using CMR-measured RV volume data. 


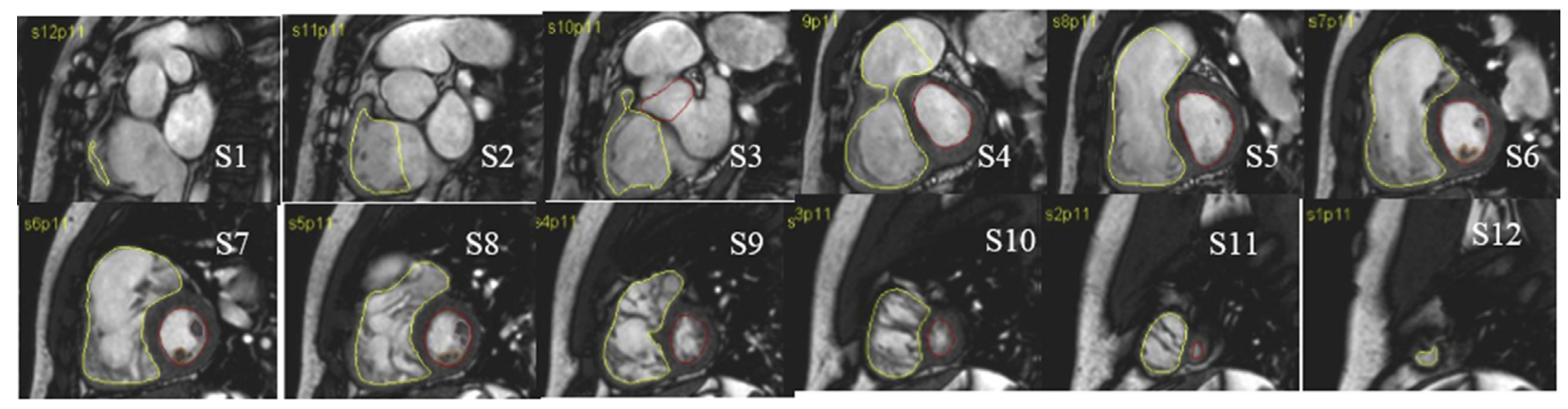

A

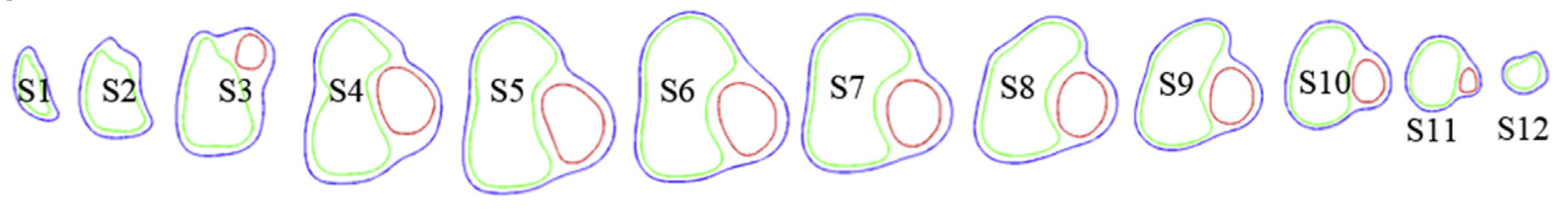

B
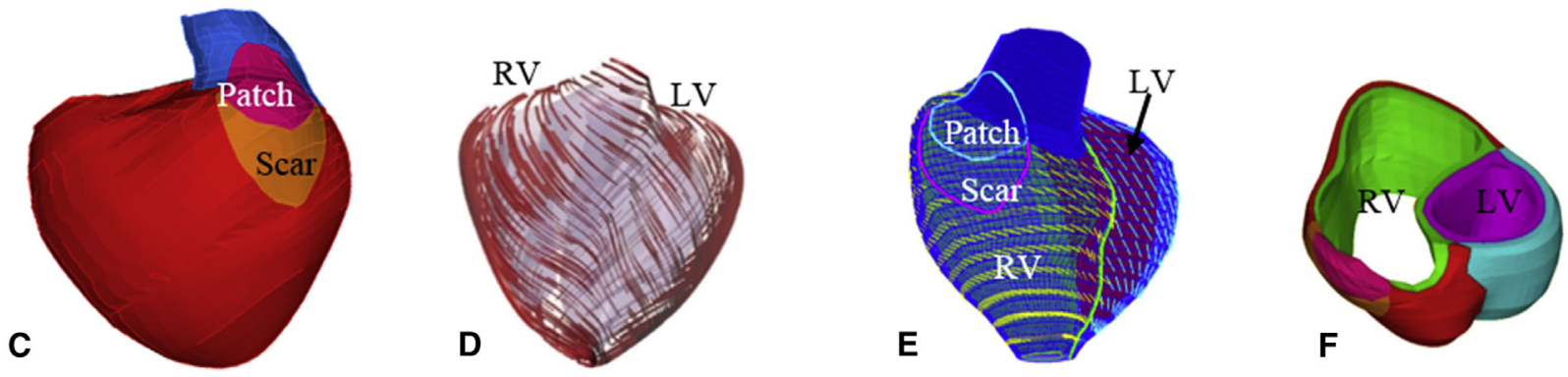

FIGURE 1. CMR-based model construction process. A, CMR image of a patient, end of systole. B, Segmented contours. C, Reconstructed 3-dimensional geometry. D and E, Fiber orientation. F, 2-layer construction. $R V$, Right ventricle; $L V$, left ventricle.

The governing equations for all material models were as follows:

$$
\begin{gathered}
\rho \mathrm{v}_{\mathrm{i}, \mathrm{tt}}=\sigma_{\mathrm{ij}, \mathrm{j}}, \quad \mathrm{i}, \mathrm{j}=1,2,3 ; \text { sum over } \mathrm{j}, \\
\varepsilon_{\mathrm{ij}}=\left(\mathrm{v}_{\mathrm{i}, \mathrm{j}}+\mathrm{v}_{\mathrm{j}, \mathrm{i}}+\mathrm{v}_{\alpha, \mathrm{i}} \mathrm{v}_{\alpha, \mathrm{j}}\right) / 2, \quad \mathrm{i}, \mathrm{j}, \alpha=1,2,3,
\end{gathered}
$$

where $\boldsymbol{\sigma}$ is the stress tensor, $\boldsymbol{\varepsilon}$ is the strain tensor, $\mathbf{v}$ is displacement, and $\rho$ is material density. The normal stress was assumed to be zero on the outer (epicardial) RV/LV surface and equal to the pressure conditions imposed on the inner (endocardial) RV/LV surfaces. Structure-only RV/LV models were used to optimize model computing time. These models provided RV volume, EFs, and RV stress/strain values for analysis. RV pressure was obtained from pre-PVR cardiac catheterization.

The RV and LV materials were assumed to be hyperelastic, anisotropic, and nearly incompressible. The patch and scar materials were assumed to be hyperelastic, isotropic, and nearly incompressible. The nonlinear Mooney-Rivlin model was used to describe the nonlinear anisotropic and isotropic material properties. The strain energy function for the isotropic modified Mooney-Rivlin model is given by Tang and colleagues ${ }^{15-17}$ :

$$
\mathrm{W}=\mathrm{c}_{1}\left(\mathrm{I}_{1}-3\right)+\mathrm{c}_{2}\left(\mathrm{I}_{2}-3\right)+\mathrm{D}_{1}\left[\exp \left(\mathrm{D}_{2}\left(\mathrm{I}_{1}-3\right)\right)-1\right],
$$

where $I_{1}$ and $I_{2}$ are the first and second strain invariants given by,

$$
\mathrm{I}_{1}=\sum \mathrm{C}_{i i}, \mathrm{I}_{2}=1 / 2\left[\mathrm{I}_{1}^{2}-\mathrm{C}_{\mathrm{ij}} \mathrm{C}_{\mathrm{ij}}\right]
$$

$\mathrm{C}=\left[\mathrm{C}_{\mathrm{ij}}\right]=\mathrm{X}^{\mathrm{T}} \mathrm{X}$ is the right Cauchy-Green deformation tensor, $\mathrm{X}=\left[\mathrm{X}_{\mathrm{ij}}\right]=\left[\partial \mathrm{x}_{\mathrm{i}} / \partial \mathrm{a}_{\mathrm{j}}\right],\left(\mathrm{x}_{\mathrm{i}}\right)$ is the current position, $\left(\mathrm{a}_{\mathrm{i}}\right)$ is the original position, and $c_{i}$ and $D_{i}$ are material parameters chosen to match experimental measurements. ${ }^{15,22}$ The parameter values are as follows: Scar, $\mathrm{c}_{1}=19.23 \mathrm{kPa}, \mathrm{c}_{2}=0, \mathrm{D}_{1}=19.23 \mathrm{kPa}, \mathrm{D}_{2}=9.0 ;$ Patch, $\mathrm{c}_{1}=38.45 \mathrm{kPa}, \mathrm{c}_{2}=0, \mathrm{D}_{1}=38.45 \mathrm{kPa}, \mathrm{D}_{2}=9.0$.

The strain energy function for the anisotropic modified Mooney-Rivlin model anisotropic model was obtained by adding an additional anisotropic term in Equation $(4)^{16,17}$ :

$$
\begin{aligned}
\mathrm{W}= & \mathrm{c}_{1}\left(\mathrm{I}_{1}-3\right)+\mathrm{c}_{2}\left(\mathrm{I}_{2}-3\right)+\mathrm{D}_{1}\left[\exp \left(\mathrm{D}_{2}\left(\mathrm{I}_{1}-3\right)\right)-1\right] \\
& +\mathrm{K}_{1} /\left(2 \mathrm{~K}_{2}\right) \exp \left[\mathrm{K}_{2}\left(\mathrm{I}_{4}-1\right)^{2}-1\right],
\end{aligned}
$$

where $I_{4}=\mathrm{C}_{\mathrm{ij}}\left(\mathbf{n}_{\mathrm{f}}\right)_{\mathrm{i}}\left(\mathbf{n}_{\mathrm{f}}\right)_{\mathrm{j}}, \mathrm{C}_{\mathrm{ij}}$ is the Cauchy-Green deformation tensor, $\mathbf{n}_{\mathrm{f}}$ is the fiber direction, and $\mathrm{K}_{1}$ and $\mathrm{K}_{2}$ are material constants. By choosing $\mathrm{c}_{1}=0.35 \mathrm{kPa}, \mathrm{c}_{2}=0, \mathrm{D}_{1}=0.063 \mathrm{kPa}, \mathrm{D}_{2}=5.3, \mathrm{~K}_{1}=1.91 \mathrm{kPa}$, $\mathrm{K}_{2}=6.00$, it was shown that stress-strain curves derived from Equation (5) agreed well with the stress-strain curves from the anisotropic (transversely isotropic) strain-energy function with respect to the local fiber direction given by McCulloch and colleagues. ${ }^{13}$

$$
\mathrm{W}=\frac{C}{2}\left(\mathrm{e}^{\mathrm{Q}}-1\right),
$$

$$
\mathrm{Q}=b_{1} E_{f f}^{2}+b_{2}\left(E_{c c}^{2}+E_{r r}^{2}+E_{c r}^{2}+E_{r c}^{2}\right)+b_{3}\left(E_{f c}^{2}+E_{c f}^{2}+E_{f r}^{2}+E_{r f}^{2}\right)
$$


where $E_{f f}$ is fiber strain, $E_{c c}$ is cross-fiber in-plane strain, $E_{r r}$ is radial strain, and $E_{c n}, E_{f}$, and $E_{f c}$ are the shear components in their respective coordinate planes. $C, b_{1}, b_{2}$, and $b_{3}$ are parameters to be chosen to fit patient data. Time-dependent parameter values $\left(\mathrm{c}_{1}(\mathrm{t}), \mathrm{D}_{1}(\mathrm{t})\right)$, and $C(\mathrm{t})$ in Equations (5) and (6) were chosen to fit the CMR-measured RV volume data for each patient to obtain patient-specific material models (Figure E2). Active contraction and expansion of myocardium were modeled by material stiffening and softening in our model reflected in the change of $\left(c_{1}(t), D_{1}(t)\right)$, and $\mathrm{C}(\mathrm{t})$ values in Equations (5) and (6).

Because patient-specific fiber orientation data were not available, we chose to construct a 2-layer RV/LV model and set fiber orientation angles using the fiber angles published by Hunter and colleagues ${ }^{14}$ (Figure 1) and available human data. ${ }^{14,23}$ Figure 1 shows epicardial and endocardial fiber layers from human and pig hearts and how the 2-layer RV/LV model was constructed. ${ }^{15-17}$

\section{Solution Methods and Simulation Procedures}

The anisotropic RV/LV computational models were constructed for the 16 patients, and the models were solved by ADINA (ADINA R\&D, Watertown, Mass) using unstructured finite elements and the Newton-Raphson iteration method. Stress/strain distributions were computed. Because stress and strain are tensors, for simplicity, maximum principal stress and strain were used and referred to as stress and strain in this article. Figure E3 shows stress/strain plots from a cut surface of an RV model, illustrating stress/ strain distribution patterns at the beginning of ejection and beginning of filling phases.

\section{Wall Thickness and Curvature Calculations}

In each CMR data set, every slice was divided into 4 quarters, each with equal inner wall circumferential length. Ventricular WT, circumferential curvature, longitudinal curvature, and stress/strain were calculated at all nodal points (100 points per slice, 25 points per quarter). Averaging their quantities over the 25 points in each quarter provided the "quarter" values of these parameters.

Circumferential curvature $\left(\kappa_{\mathrm{c}}\right)$ at each point on an $\mathrm{RV}$ inner contour was calculated using

$$
\kappa_{c}=\frac{x^{\prime} y^{\prime \prime}-x^{\prime \prime} y^{\prime}}{\left(x^{\prime 2}+y^{\prime 2}\right)^{3 / 2}}
$$

where the contour was a planar curve, $\mathrm{x}$ and $\mathrm{y}$ are treated as an arc function, and the derivatives were evaluated using neighboring points on the contour. Longitudinal curvature $(\kappa)$ at each point on an RV inner contour was calculated using analysis. Logistic regression analysis was used to identify pre-PVR parameters that best predicted the primary outcome- $-\mathrm{RV}$ EF response to PVR. The sensitivity and specificity of these parameters and their area under the receiver operating characteristic (ROC) curve were determined.

To examine the validity of the logistic regression model, a 2-fold crossvalidation procedure was used for the model fitting and prediction procedure. Specifically, we randomly selected 8 of 16 patients as training data to fit a model that reached the best agreement between predictors' values and the binary group outcome (optimal vs suboptimal RV EF response to PVR). The data of the remaining patients (test data) were then fed into the model to calculate the probabilities of their outcome group status. The training and test data were then interchanged, and the same procedure was followed to complete a 2 -fold cross-validation. To stabilize the result, we repeated the 2-fold cross-validation 100 times (each with a random partition of training and testing groups). The probabilities of group assignments from all cross-validation procedures were then combined to calculate the final prediction values. All statistical tests were 2-sided. Data analysis was performed using $\mathrm{R}$ package. ${ }^{24}$

\section{RESULTS}

\section{Agreement Between Cardiac Magnetic Resonance Data and Computational Modeling}

The stress-stretch curves and parameter values of the RV/ LV tissues at the beginning of ejection and beginning of filling in a representative model are shown in Figure E2, $A$. The imposed RV pressure conditions and computational $\mathrm{RV}$ volume data used in the same model are presented in Figure E2, B. Good agreement between computational and CMR-measured volume data was found (error $<2 \%$ ), as exemplified in Figure E2, $C$.

\section{Right Ventricular Ejection Fraction Change Correlated Negatively With Right Ventricular Stress and Volume}

Table 1 summarizes the RV size, geometric parameters, and stress/strain data in the study patients. Correlation analyses were performed to determine whether changes in RV EF from pre- to post-PVR were associated with RV size (volumes and WT), geometry (circumferential and longitudinal curvatures), or stress/strain data. In this cohort, RV EF change corre-

$$
\kappa=\frac{\sqrt{\left(z^{\prime \prime}(t) y^{\prime}(t)-y^{\prime \prime}(t) z^{\prime}(t)\right)^{2}+\left(x^{\prime \prime}(t) z^{\prime}(t)-z^{\prime \prime}(t) x^{\prime}(t)\right)^{2}+\left(y^{\prime \prime}(t) x^{\prime}(t)-x^{\prime \prime}(t) y^{\prime}(t)\right)^{2}}}{\left(x^{\prime 2}(t)+y^{\prime 2}(t)+z^{\prime 2}(t)\right)^{3 / 2}}
$$

where the longitudinal curve is given by $X=(x(t), y(t), z(t))$. The derivatives were evaluated using points from neighboring slices vertically below and above the point being considered. Interpolations were used to obtain vertical neighboring points from adjacent slices. One-sided formulas were used for the top and bottom slices.

\footnotetext{
Statistical Analysis

Continuous variables (RV volumes, WT, circumferential and longitudinal curvatures, and stress and strain values) were summarized as mean \pm standard deviation or median (range) and compared between the outcome groups using an unpaired Student $t$ test. Associations between pre-PVR RV parameters and the outcome (change in RV EF) were explored using Pearson correlation
}

lated negatively with stress $(r=-0.56, P=.025)$ (Figure E4, $A)$ and pre-PVR RV end-diastole volume $(r=-0.60$, $P=.015$ ) (Figure E4, $B$ ), but did not correlate with WT, circumferential curvature, longitudinal curvature, or strain.

\section{Group Comparison: Right Ventricular Stress Higher in Group 2 than in Group 1}

Table 2 summarizes the comparison of RV WT, circumferential curvature, longitudinal curvature, volume, and stress and strain values between the outcome groups at the 
TABLE 1. Summary of geometric and stress/strain parameters averaged in each patient at onset of ejection and their correlations with right ventricular ejection fraction change

\begin{tabular}{|c|c|c|c|c|c|c|c|}
\hline Group & $\Delta \mathrm{EF}(\%)$ & WT (cm) & $\begin{array}{l}\text { Circumferential } \\
\text { curvature }(1 / \mathrm{cm})\end{array}$ & $\begin{array}{c}\text { Longitudinal } \\
\text { curvature (1/cm) }\end{array}$ & RV EDV $(\mathbf{m L})$ & Stress (kPa) & Strain \\
\hline \multicolumn{8}{|l|}{ Group 1} \\
\hline & 1.4 & 0.39 & 0.47 & 1.24 & 406.9 & 56.9 & 0.29 \\
\hline & -3.4 & 0.34 & 0.39 & 0.77 & 328.8 & 65.3 & 0.43 \\
\hline & -2.6 & 0.65 & 0.37 & 1.01 & 408.8 & 41.0 & 0.33 \\
\hline & -2.9 & 0.49 & 0.54 & 1.54 & 364.6 & 64.1 & 0.36 \\
\hline & 4.0 & 0.47 & 0.43 & 0.96 & 323.3 & 82.4 & 0.44 \\
\hline & 5.6 & 0.48 & 0.50 & 1.20 & 204.0 & 61.9 & 0.48 \\
\hline & 6.6 & 0.42 & 0.53 & 1.84 & 193.7 & 33.5 & 0.46 \\
\hline & 2.0 & 0.51 & 0.53 & 1.85 & 188.3 & 42.0 & 0.40 \\
\hline Mean $\pm \mathrm{SD}$ & $1.34 \pm 3.9$ & $0.47 \pm 0.09$ & $0.47 \pm 0.07$ & $1.3 \pm 0.4$ & $302.3 \pm 93.9$ & $55.9 \pm 16.1$ & $0.40 \pm 0.07$ \\
\hline \multicolumn{8}{|l|}{ Group 2} \\
\hline & -18.0 & 0.48 & 0.42 & 0.91 & 385.1 & 172.1 & 0.66 \\
\hline & -8.4 & 0.41 & 1.34 & 1.32 & 204.2 & 82.9 & 0.49 \\
\hline & -15.2 & 0.80 & 0.36 & 0.59 & 665.1 & 82.4 & 0.23 \\
\hline & -7.0 & 0.71 & 0.44 & 0.72 & 334.8 & 83.1 & 0.42 \\
\hline & -5.0 & 0.45 & 0.46 & 0.97 & 277.2 & 191.7 & 0.66 \\
\hline & -9.5 & 0.43 & 0.65 & 1.60 & 365.0 & 65.4 & 0.44 \\
\hline & -12.3 & 0.46 & 0.44 & 1.23 & 299.0 & 154.3 & 0.51 \\
\hline & -13.4 & 0.59 & 0.33 & 1.25 & 571.1 & 76.2 & 0.34 \\
\hline Mean $\pm \mathrm{SD}$ & $-11.1 \pm 4.4$ & $0.54 \pm 0.14$ & $0.56 \pm 0.33$ & $1.07 \pm 0.34$ & $387.7 \pm 154.8$ & $113.5 \pm 50.4$ & $0.47 \pm 015$ \\
\hline$* R$ value & & -0.35 & 0.01 & 0.41 & -0.60 & -0.56 & -0.11 \\
\hline${ }^{*} P$ value & & .18 & .97 & .11 & .015 & .025 & .68 \\
\hline
\end{tabular}

$\Delta E F$, Right ventricular ejection fraction change; $W T$, wall thickness; $R V$, right ventricle; $E D V$, end-diastolic volume; $S D$, standard deviation. $* R$ and $P$ values are for the correlations between change in RV EF and geometric and stress/strain data.

onsets of ejection and filling. At the onset of ejection (maximal volume and pressure), mean RV stress was $103 \%$ higher in group 2 compared with group 1 $(113.5 \pm 50.4 \mathrm{kPa}$ vs $55.9 \pm 16.12 \mathrm{kPa} ; P=.008)$. Furthermore, stress was the only parameter that showed significant differences between the 2 groups. Similar findings were noted at the onset of filling with substantially lower values (Table 2).

\section{Right Ventricular Stress is the Best Predictor for the Outcome Group Category Using Median as Threshold}

By using the medians of the parameters shown in Table 2 as threshold values, we examined whether the preoperative measurements in each patient predicted the patient's group category. For example, the median RV stress at onset of ejection for the 16 patients was $70.8 \mathrm{kPa}$. If a patient's preoperative mean stress value was lower, the patient was predicted to belong to group 1 , and if the measurement was higher, the patient was predicted to belong to group 2 . If the prediction matches the actual group assignment, the prediction was considered "true." Otherwise, the prediction was considered "false." The results of the analysis are shown in Table 3. By using the values noted in the example, group assignments based on the median value of RV stress were correct in 14 of the 16 cases $(87.5 \%, 95 \%$ confidence interval, 62.5-100). Other parameters showed lower rates of correct group assignment.

TABLE 2. Comparison of right ventricular volumes, geometric parameters, and stress/strain between group 1 and group 2 at onset of ejection and onset of filling

\begin{tabular}{|c|c|c|c|c|c|c|}
\hline \multirow[b]{2}{*}{ Parameter/Predictor } & \multicolumn{3}{|c|}{$\begin{array}{c}\text { Beginning of ejection } \\
\text { (maximal volume and pressure) }\end{array}$} & \multicolumn{3}{|c|}{$\begin{array}{c}\text { Beginning of filling } \\
\text { (minimal volume and pressure) }\end{array}$} \\
\hline & Group 1 & Group 2 & $P$ value & Group 1 & Group 2 & $P$ value \\
\hline $\mathrm{RV}$ volume $(\mathrm{mL})$ & $302.3 \pm 93.9$ & $387.7 \pm 154.8$ & .20 & $180.0 \pm 67.0$ & $228.4 \pm 121.3$ & .34 \\
\hline $\mathrm{WT}(\mathrm{cm})$ & $0.46 \pm 0.09$ & $0.54 \pm 0.14$ & .25 & $0.51 \pm 0.10$ & $0.61 \pm 0.17$ & .21 \\
\hline Circumferential curvature $(1 / \mathrm{cm})$ & $0.47 \pm 0.07$ & $0.55 \pm 0.33$ & .50 & $0.59 \pm 0.10$ & $0.67 \pm 0.35$ & .55 \\
\hline Longitudinal curvature $(1 / \mathrm{cm})(1 / \mathrm{cm})$ & $1.30 \pm 0.40$ & $1.07 \pm 0.34$ & .24 & $1.35 \pm 0.53$ & $1.08 \pm 0.31$ & .24 \\
\hline Stress $(\mathrm{kPa})$ & $55.9 \pm 16.1$ & $113.5 \pm 50.4$ & .008 & $4.15 \pm 3.24$ & $10.49 \pm 5.81$ & .017 \\
\hline Strain & $0.40 \pm 0.07$ & $0.47 \pm 0.14$ & .25 & $0.04 \pm 0.04$ & $0.08 \pm 0.06$ & .20 \\
\hline
\end{tabular}

Data are based on quarter mean values (see "Materials and Methods" for details). Values are expressed as mean \pm standard deviation. $R V$, Right ventricular; $W T$, wall thickness. 
TABLE 3. True or false prediction results using the mean parameter values to predict patient's group

\begin{tabular}{|c|c|c|c|c|c|c|}
\hline Patient & RV EDV (mL) & WT (cm) & $\begin{array}{l}\text { Circumferential } \\
\text { curvature }(1 / \mathrm{cm})\end{array}$ & $\begin{array}{c}\text { Longitudinal } \\
\text { curvature }(1 / \mathrm{cm})\end{array}$ & Stress & Strain \\
\hline $\mathrm{P} 1$ & $406.9 \mathrm{~F}$ & $0.39 \mathrm{~T}$ & $0.47 \mathrm{~T}$ & $1.24 \mathrm{~T}$ & $56.9 \mathrm{~T}$ & $0.29 \mathrm{~T}$ \\
\hline $\mathrm{P} 2$ & $328.8 \mathrm{~T}$ & $0.34 \mathrm{~T}$ & $0.39 \mathrm{~F}$ & $0.77 \mathrm{~F}$ & $65.3 \mathrm{~T}$ & $0.43 \mathrm{~T}$ \\
\hline P3 & $408.8 \mathrm{~F}$ & $0.65 \mathrm{~F}$ & $0.37 \mathrm{~F}$ & $1.01 \mathrm{~F}$ & $41.0 \mathrm{~T}$ & $0.33 \mathrm{~T}$ \\
\hline P4 & $364.6 \mathrm{~F}$ & $0.49 \mathrm{~F}$ & $0.54 \mathrm{~T}$ & $1.54 \mathrm{~T}$ & $64.1 \mathrm{~T}$ & $0.36 \mathrm{~T}$ \\
\hline P5 & $323.3 \mathrm{~T}$ & $0.47 \mathrm{~T}$ & $0.43 \mathrm{~F}$ & $0.96 \mathrm{~F}$ & $82.4 \mathrm{~F}$ & $0.44 \mathrm{~F}$ \\
\hline P6 & $204.0 \mathrm{~T}$ & $0.48 \mathrm{~F}$ & $0.50 \mathrm{~T}$ & $1.20 \mathrm{~F}$ & $61.9 \mathrm{~T}$ & $0.48 \mathrm{~F}$ \\
\hline P7 & $193.7 \mathrm{~T}$ & $0.43 \mathrm{~T}$ & $0.53 \mathrm{~T}$ & $1.84 \mathrm{~T}$ & $33.5 \mathrm{~T}$ & $0.46 \mathrm{~F}$ \\
\hline P8 & $188.3 \mathrm{~T}$ & $0.51 \mathrm{~F}$ & $0.53 \mathrm{~T}$ & $1.85 \mathrm{~T}$ & $42.0 \mathrm{~T}$ & $0.40 \mathrm{~T}$ \\
\hline P9 & $385.1 \mathrm{~T}$ & $0.48 \mathrm{~T}$ & $0.42 \mathrm{~T}$ & $0.91 \mathrm{~T}$ & $172.1 \mathrm{~T}$ & $0.66 \mathrm{~T}$ \\
\hline P10 & $204.2 \mathrm{~F}$ & $0.41 \mathrm{~F}$ & $1.34 \mathrm{~F}$ & $1.32 \mathrm{~F}$ & $82.9 \mathrm{~T}$ & $0.49 \mathrm{~T}$ \\
\hline P11 & $665.1 \mathrm{~T}$ & $0.80 \mathrm{~T}$ & $0.36 \mathrm{~T}$ & $0.59 \mathrm{~T}$ & $82.4 \mathrm{~T}$ & $0.23 \mathrm{~F}$ \\
\hline P12 & $334.8 \mathrm{~T}$ & $0.71 \mathrm{~T}$ & $0.44 \mathrm{~T}$ & $0.72 \mathrm{~T}$ & $83.1 \mathrm{~T}$ & $0.42 \mathrm{~F}$ \\
\hline P13 & $277.2 \mathrm{~F}$ & $0.46 \mathrm{~F}$ & $0.46 \mathrm{~F}$ & $0.97 \mathrm{~T}$ & $191.7 \mathrm{~T}$ & $0.66 \mathrm{~T}$ \\
\hline P14 & $365.0 \mathrm{~T}$ & $0.43 \mathrm{~F}$ & $0.65 \mathrm{~F}$ & $1.60 \mathrm{~F}$ & $65.4 \mathrm{~F}$ & $0.44 \mathrm{~T}$ \\
\hline P15 & $299.0 \mathrm{~F}$ & $0.46 \mathrm{~F}$ & $0.44 \mathrm{~T}$ & $1.24 \mathrm{~F}$ & $154.3 \mathrm{~T}$ & $0.51 \mathrm{~T}$ \\
\hline P16 & $571.1 \mathrm{~T}$ & $0.59 \mathrm{~T}$ & $0.33 \mathrm{~T}$ & $1.25 \mathrm{~F}$ & $76.2 \mathrm{~T}$ & $0.34 \mathrm{~F}$ \\
\hline Median & 331.8 & 0.47 & 0.45 & 1.21 & 70.8 & 0.43 \\
\hline True prediction & $62.5 \%$ & $50 \%$ & $62.5 \%$ & $50 \%$ & $87.5 \%$ & $62.5 \%$ \\
\hline CI & $(37.5-87.5)$ & $(37.5-87.5)$ & $(12.5-75)$ & $(37.5-87.5)$ & $(62.5-100)$ & $(37.5-87.5)$ \\
\hline
\end{tabular}

The median values were used as the thresholds for group assignments. $R V$, Right ventricular; $E D V$, end-diastolic volume; $W T$, wall thickness; $F$, false; $T$, true; $C I$, Confidence interval.

\section{Right Ventricular Stress has the Best Prediction Accuracy of the 6 Parameters Using the Logistic Regression Model}

The logistic regression method was applied to all 63 possible combinations of the 6 candidate predictors WT, circumferential curvature, longitudinal curvature, RV volume, stress, and strain to calculate their prediction accuracy for the patient's group category. Table 4 shows the 6 best combinations (of 63) of RV parameters that correctly assigned patients to their ultimate outcome group. Pre-PVR RV stress was the best single predictor among the 6 individual parameters with an area under the ROC curve of 0.819 . The second best single predictor was strain with an area under the ROC curve of 0.544 . The best combination of parameters included WT + circumferential curvature $+\mathrm{RV}$ volume + strain an area under the ROC curve of 0.848 . Among all combinations of parameters, stress was the best predictor on the basis of sensitivity and specificity.

\section{DISCUSSION}

This study provides proof of concept that computational modeling based on patient-specific CMR image data can be used to predict RV response to PVR in patients with repaired TOF. Although computational modeling has been used in selecting congenital cardiac anomalies such as the Fontan circulation and repaired coarctation, ${ }^{25,26}$ it has not been used to evaluate the RV in patients with congenital

TABLE 4. Prediction sensitivity, specificity, area under the curve values, and right ventricular parameters for outcome group prediction by logistic regression method

\begin{tabular}{|c|c|c|c|c|c|c|c|c|}
\hline Parameter & $\begin{array}{c}\text { Probability } \\
\text { cutoffs }\end{array}$ & Sensitivity & Specificity & $\begin{array}{c}\text { Sensitivity }+ \\
\text { specificity }\end{array}$ & $\mathbf{A U C}$ & AUC average & $95 \% \mathbf{C I}$ & Rank \\
\hline $\mathrm{WT}+$ circumferential curvature + volume + strain & 1.000 & 0.75 & 0.78 & 1.53 & 0.840 & 0.848 & $0.838-0.850$ & 1 \\
\hline Stress & 0.626 & 0.80 & 0.85 & 1.65 & 0.822 & 0.819 & $0.818-0.822$ & 2 \\
\hline Circumferential curvature + volume + stress & 1.000 & 0.78 & 0.74 & 1.51 & 0.802 & 0.806 & $0.803-0.811$ & 3 \\
\hline $\mathrm{WT}+$ circumferential curvature + stress & 0.632 & 0.86 & 0.60 & 1.46 & 0.753 & 0.796 & $0.791-0.808$ & 4 \\
\hline $\mathrm{WT}+$ circumferential curvature + volume + stress & 0.001 & 0.89 & 0.56 & 1.45 & 0.743 & 0.794 & $0.792-0.802$ & 5 \\
\hline WT + longitudinal curvature + stress & 0.073 & 0.71 & 0.68 & 1.39 & 0.735 & 0.775 & $0.770-0.778$ & 6 \\
\hline Strain & 0.494 & 0.64 & 0.55 & 1.19 & 0.542 & 0.544 & $0.538-0.558$ & \\
\hline Volume & 0.276 & 0.96 & 0.14 & 1.10 & 0.487 & 0.513 & $0.506-0.519$ & \\
\hline Longitudinal curvature & 0.849 & 0.19 & 0.94 & 1.13 & 0.558 & 0.508 & $0.504-0.515$ & \\
\hline WT & 0.444 & 0.78 & 0.25 & 1.03 & 0.407 & 0.489 & $0.478-0.499$ & \\
\hline Circumferential curvature & 0.459 & 0.84 & 0.24 & 1.08 & 0.408 & 0.410 & $0.407-0.416$ & \\
\hline
\end{tabular}

AUC average and $95 \%$ CI are based on 200 rounds of 10 repeats. AUC, Area under the curve; $C I$, confidence interval; WT, wall thickness. 
heart disease affecting the right heart, such as repaired TOF. With the use of patient-specific CMR image data, we developed 2-layer anisotropic models of the RVs and LVs with material properties of the RV myocardium derived from in vitro biaxial testing. These complex computational models have provided new insights into RV morphology (volumes and WT), geometry (circumferential and longitudinal curvatures), and mechanics (RV stress and strain) in repaired TOF.

With the rapidly increasing number of late survivors of TOF repair, surgical management of patients with RV dysfunction has become a major clinical challenge. The wide variability in clinical status, variations of $\mathrm{RV}$ morphology, regurgitation at both tricuspid and pulmonary valves, extent of RV dilatation, scarring, and dysfunction at the time of presentation have resulted in disparate surgical results with pulmonary valve insertion. ${ }^{1}$ To our knowledge, this is the first report providing evidence that RV stress before PVR may be used as a predictor of RV functional response to pulmonary valve implantation.

The current study adds computational modeling as a new investigative tool and stress/strain as new potential predictors for postoperative functional outcomes after PVR. It should be noted that stress/strain calculations depend heavily on data available and model assumptions. However, comparative studies can still provide meaningful results as long as the same data and modeling standard were applied to all patients. The results of this computational analysis study are intriguing. Specifically, RV stress stands out as the best predictor of RV function response to PVR among the 6 parameters examined. From a pathophysiologic perspective, these findings are plausible given that stress more accurately reflects the functional status of the myocardium compared with EF, which is a global parameter of pump function influenced by loading conditions. From a clinical perspective, most published criteria for PVR have focused on RV volume-based parameters and EF. However, the results of this study suggest that RV stress may be more helpful in identifying the likelihood of functional recovery of the RV, thus informing the decision to recommend PVR rather than relying on $\mathrm{RV}$ volumes alone. We recognize that our preliminary findings and their potential clinical implications require validation by large-scale studies. However, it is worth noting that construction of patient-specific 2-layer models with fiber orientation is labor-intensive and that with the current state of computer technology, it takes approximately 1 month to generate each model. Refinement of the model-building technique and advances in computer science will be essential for making the process less labor intensive and more clinically applicable.

\section{Study Limitations}

Several limitations of this study are worth noting. The small sample size, which reflects the proof-of-concept nature of this study, results in limited statistical power. Furthermore, the patients included in this study may not reflect the general population of patients with repaired TOF because they were drawn from a larger sample of individuals undergoing PVR. Another reason for the small sample size is the extensive amount of time required for constructing each computational model ( $\sim 1$ month). As noted earlier, future work will aim at improving the model-building process to make it more clinically applicable.

Several improvements can enhance our models in the future for better accuracy and applicability, including the following: (1) The addition of valve mechanics to the model may improve accuracy with regard to the timing of valve opening and closing and allow incorporation of tricuspid and pulmonary valve regurgitation. (2) The addition of fluid-structure interaction can be helpful to obtain both flow and structural stress/strain information for a more comprehensive mechanical analysis. Fluid-structure interaction models could provide flow information that is important for RV remodeling and tissue regeneration process. (3) The addition of regional tissue mechanical properties and patient-specific fiber orientations will be a desirable addition for improved accuracy of our models. (4) Development of methods to model active contraction by adding active stress and techniques for adjusting zero-stress fiber length will be helpful. (5) Development of multiscale models including organ, cell, and gene investigations will be helpful. The latter will allow mechanical conditions obtained at the organ level to be integrated with information from the cellular and genetic levels to explore mechanisms of right heart failure and its treatment.

\section{CONCLUSIONS}

In this proof-of-concept study of 16 patients with repaired TOF undergoing PVR, computational modeling based on patient-specific CMR images demonstrated that among several morphologic, geometric, and RV mechanic parameters, stress was the best predictor of RV response to pulmonary valve implantation. These findings provide a basis for future studies aimed at validation of these results in larger groups of patients and further refinements of the computational modeling technique to improve its accuracy and practicality.

\section{Conflict of Interest Statement}

T.G. is a consultant to Medtronic Inc. All other authors have nothing to disclose with regard to commercial support.

\section{References}

1. Ooi A, Moorjani N, Baliulis G, Keeton BR, Salmon AP, Monro JL, et al. Medium term outcome for infant repair in tetralogy of Fallot: indicators for timing of surgery. Eur J Cardiothorac Surg. 2006;30:917-22. 
2. Chiu SN, Wang JK, Chen HC, Lin MT, Wu ET, Chen CA, et al. Long-term survival and unnatural deaths of patients with repaired tetralogy of Fallot in an Asian cohort. Circ Cardiovasc Qual Outcomes. 2012;5:120-5.

3. McKenzie ED, Khan MS, Dietzman TW, Guzmán-Pruneda FA, Samayoa AX, Liou A, et al. Surgical pulmonary valve replacement: a benchmark for outcomes comparisons. J Thorac Cardiovasc Surg. 2014;148:1450-3.

4. Waien SA, Liu PP, Ross BL, Williams WG, Webb GD, McLaughlin PR. Serial follow-up of adults with repaired tetralogy of Fallot. J Am Coll Cardiol. 1992; 20:295-300.

5. Burchill LJ, Wald RM, Harris L, Colman JM, Silversides CK. Pulmonary valve replacement in adults with repaired tetralogy of Fallot. J Thorac Cardiovasc Surg. 2011;14:92-7.

6. Therrien J, Siu SC, McLaughlin PR. Pulmonary valve replacement in adults late after repair of tetralogy of Fallot: are we operating too late? J Am Coll Cardiol. 2000;36:1670-5.

7. Meijboom FJ, Roos-Hesselink JW, McGhie JS, Spitaels SE, van Domburg RT, Utens LM, et al. Consequences of a selective approach toward pulmonary valve replacement in adult patients with tetralogy of Fallot and pulmonary regurgitation. J Thorac Cardiovasc Surg. 2008;135:50-5.

8. Vliegen HW, Van Straten A, De Roos A, Roest AA, Schoof PH, Zwinderman $\mathrm{AH}$, et al. Magnetic resonance imaging to assess the hemodynamic effects of pulmonary valve replacement in adults late after repair of tetralogy of Fallot. Circulation. 2002;106:1703-7.

9. Tweddell JS, Simpson P, Li SH, Dunham-Ingle J, Bartz PJ, Earing MG, et al. Timing and technique of pulmonary valve replacement in the patient with tetralogy of Fallot. J Thorac Cardiovasc Surg. 2012;15:27-33.

10. Geva T. Repaired tetralogy of Fallot: the roles of cardiovascular magnetic resonance in evaluating pathophysiology and for pulmonary valve replacement decision support. J Cardiovasc Magn Reson. 2011;13:9.

11. Valente A, Gauvreau K, Egidy Assenza G, Babu-Narayan SV, Schreier J, Gatzoulis M, et al. Contemporary predictors of death and sustained ventricular tachycardia in patients with repaired tetralogy of Fallot enrolled in the INDICATOR cohort. Heart. 2014;100:247-53.

12. Yang C, Tang D, Geva T, Rathod R, Yamauchi H, Gooty V, et al. Using contracting band to improve right ventricle ejection fraction for patients with repaired tetralogy of Fallot: a modeling study using patient-specific CMR-based 2-layer anisotropic models of human right and left ventricles. J Thorac Cardiovasc Surg. 2013;145:285-93.

13. McCulloch AD, Waldman L, Rogers J, Guccione JM. Large-scale finite element analysis of the beating heart. Crit Rev Biomed Eng. 1992;20:427-49.

14. Hunter PJ, Pullan AJ, Smaill BH. Modeling total heart function. Annu Rev Biomed Eng. 2003;5:147-77.
15. Tang D, Yang C, Geva T, del Nido PJ. Image-based patient-specific ventricle models with fluid-structure interaction for cardiac function assessment and surgical design optimization. Prog Pediatr Cardiol. 2010;30:51-62.

16. Tang D, Yang C, Geva T, Gaudette G, del Nido PJ. Multi-physics MRI-based two-layer fluid-structure interaction anisotropic models of human right and left ventricles with different patch materials: cardiac function assessment and mechanical stress analysis. Comput Struct. 2011;89:1059-68.

17. Tang D, Yang C, Geva T, del Nido PJ. Patient-specific MRI-based 3D FSI RV/LV/ Patch models for pulmonary valve replacement surgery and patch optimization. $J$ Biomech Eng. 2008;130:041010.

18. Tang D, Yang C, Geva T, del Nido PJ. Right ventricular local longitudinal curvature as a marker and predictor for pulmonary valve replacement surgery outcome: an initial study based on preoperative and postoperative cardiac magnetic resonance data from patients with repaired tetralogy of Fallot. J Thorac Cardiovasc Surg. 2014; 147:537-8.

19. Geva T, Gauvreau K, Powell AJ, Cecchin F, Rhodes J, Geva J, et al. Randomized trial of pulmonary valve replacement with and without right ventricular remodeling surgery. Circulation. 2010;122(11 Suppl):S201-8.

20. Sacks MS, Chuong CJ. Biaxial mechanical properties of passive right ventricular free wall myocardium. J Biomech Eng. 1993;115:202-5.

21. Billiar KL, Sacks MS. Biaxial mechanical properties of the natural and glutaraldehyde treated aortic valve cusp-Part I: Experimental results. J Biomech Eng. 2000;122:23-30.

22. Humphrey JD. Cardiovascular Solid Mechanics. New York: Springer-Verlag; 2002.

23. Sanchez-Quintana D, Anderson R, Ho SY. Ventricular myoarchitecture in tetralogy of Fallot. Heart. 1996;76:280-6.

24. R Core Team. R: A Language and Environment for Statistical Computing. Vienna, Austria: R Foundation for Statistical Computing; 2014. Available at: http://www.R-project.org. Accessed August 29, 2015.

25. Haggerty CM, Restrepo M, Tang E, de Zelicourt DA, Sundareswaran KS, Mirabella L, et al. Fontan hemodynamics from 100 patient-specific cardiac magnetic resonance studies: a computational fluid dynamics analysis. J Thorac Cardiovasc Surg. 2014;148:1481-9.

26. Olivieri LJ, de Zelicourt DA, Haggerty CM, Ratnayaka K, Cross RR, Yoganathan AP. Hemodynamic modeling of surgically repaired coarctation of the aorta. Cardiovasc Eng Technol. 2011;2:288-95.

Key Words: computational modeling, congenital heart disease, pulmonary valve replacement, right ventricle, tetralogy of Fallot 

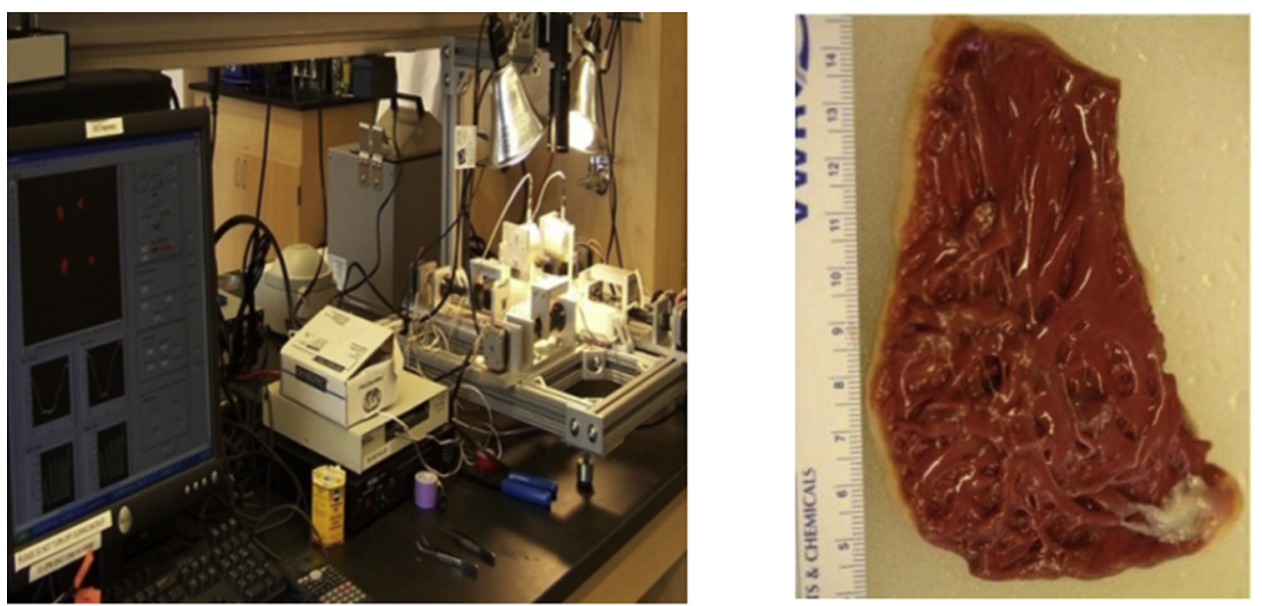

A

B

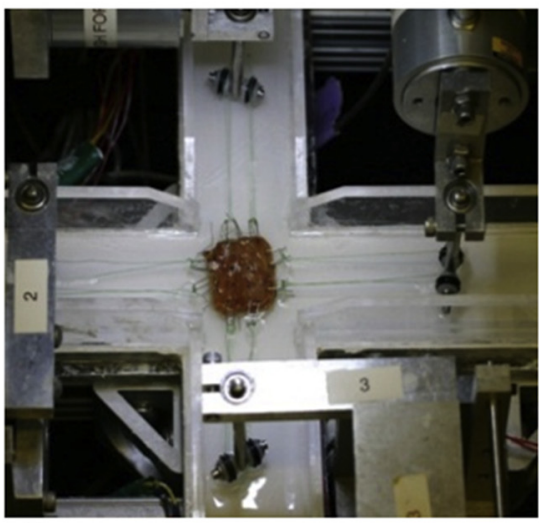

C

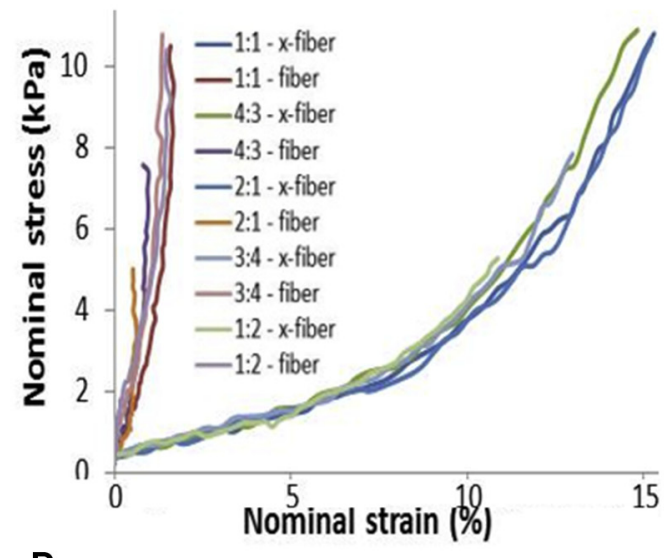

D

FIGURE E1. Biaxial mechanical testing and initial results. A, Biaxial testing apparatus. B, Human RV tissue sample. C, Tissue sample mounted for biaxial test. D, Anisotropic data from the human RV sample.
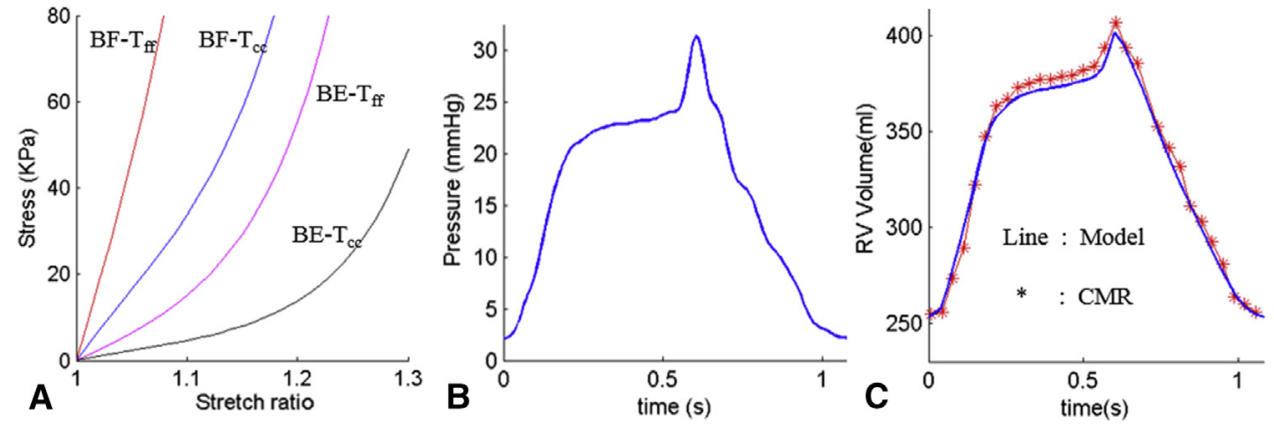

FIGURE E2. Material stress-stretch curves, pressure conditions used in the article, and computational RV volume curve matching CMR-measured data. A, Stress-stretch curves from Mooney-Rivlin anisotropic RV tissue models used in this article. Model parameter values in Equations (6) and (7) for a patient: beginning filling: $\mathrm{C}=72.16 \mathrm{kPa}, \mathrm{b}_{1}=8.7875 ; \mathrm{b}_{2}=1.7005 ; \mathrm{b}_{3}=0.7743$; beginning ejection: $\mathrm{C}=9.922 \mathrm{kPa}, \mathrm{b}_{1}=8.7875 ; \mathrm{b}_{2}=1.7005 ; \mathrm{b}_{3}=0.7743$. $\mathrm{T}_{\mathrm{ff}}$ : stress in the fiber direction; Tcc: stress in fiber circumferential direction. B, Imposed inner RV pressure. C, Model validation: computational RV volume from the fluid-structure interaction model matching CMR-measured RV volume data (error $\left.=\frac{\sum \mid C M R(i)-\text { num(i) } \mid}{\sum|C M R(i)|}=0.0167 .<2 \%\right) . B F$, Beginning filling; BE, beginning ejection; $R V$, right ventricular; $C M R$, cardiac magnetic resonance. 


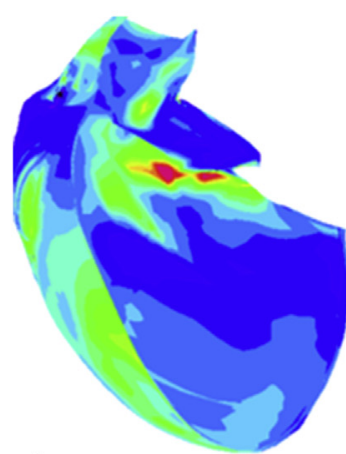

A

\begin{abstract}
B
\end{abstract}
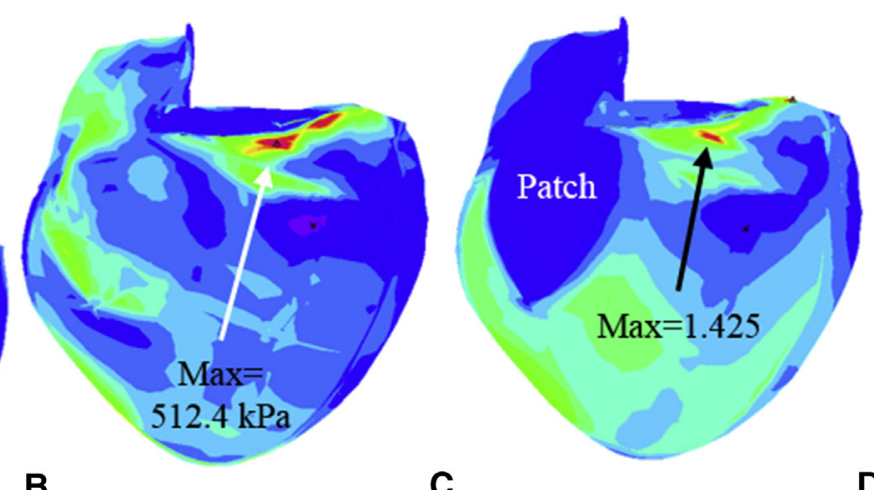

C

\section{Universal Scale}

\section{Min}

D

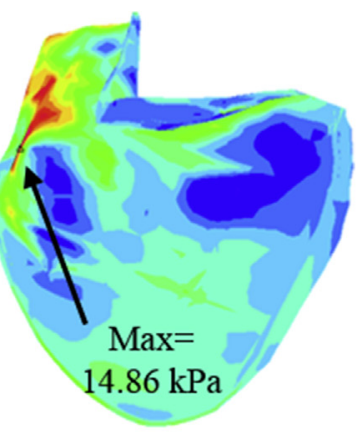

FIGURE E3. Selected cut surface, maximum principal stress and strain corresponding to end of diastole and end of systole pressure conditions. A, Position of the cut surface. B, Maximum principal stress, end of diastole. C, Maximum principal strain, end of diastole. D, Maximum principal stress, end of systole.

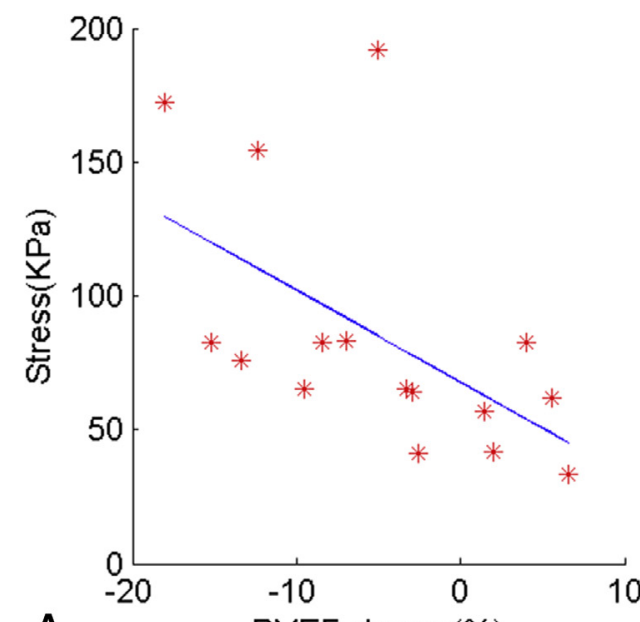

A RVEF change(\%)

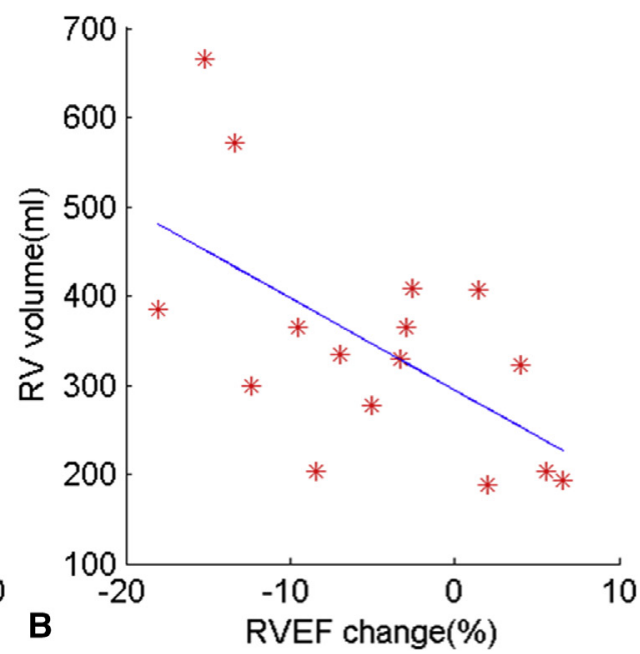

FIGURE E4. RVEF change correlated negatively with stress $(r=-0.56, P=.025)$ and pre-PVR RV volume $(r=-0.60, P=.015)$. A, Stress versus EF change. B, Pre-PVR RV volume versus EF change. $R V E F$, Right ventricular ejection fraction; $R V$, right ventricular. 
TABLE E1. Demographic and cardiac magnetic resonance data before and after pulmonary valve replacement

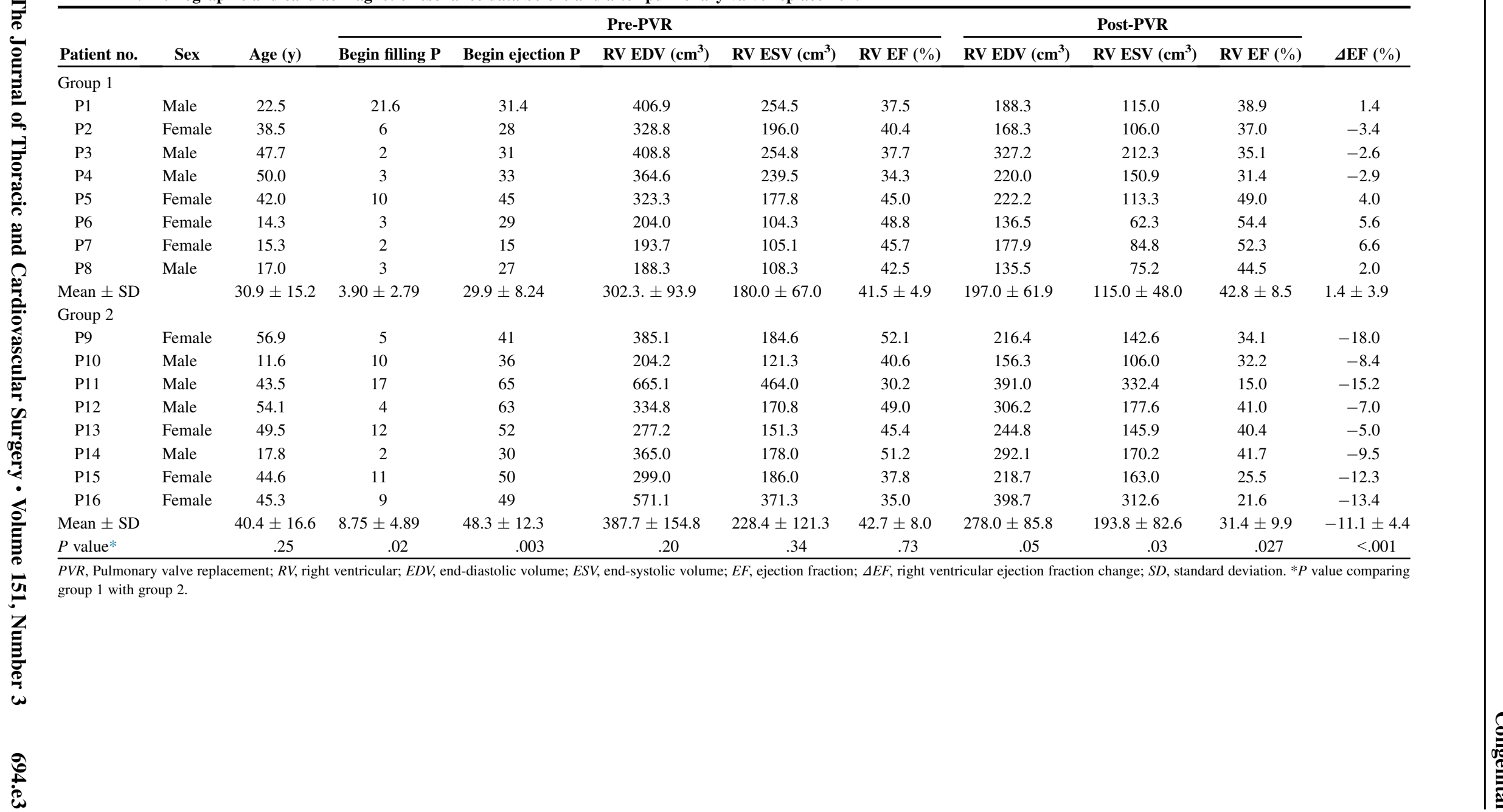

